Fernstudium

\section{Bachelor Pflegemanagement}

— Die staatlich anerkannte private Internationale Hochschule IUBH bietet den Studiengang Bachelor in Pflegemanagement (B.A.) im Fernstudium an. Mit einer Mischung aus BWL und Expertenwissen aus dem Pflegewesen bereitet der Studiengang optimal auf Führungsverantwortung in Pflegeeinrichtungen vor. Die nächsten Infoveranstaltungen zum Fernstudium Pflegemanagement finden am 8.10.2016 in Düsseldorf, am 20.10. als Onlineveranstaltung und am 29.10. in Hamburg statt.
Ganz einfach

\section{Fortbildung für Pflegedienste}

— Sie suchen eine unkomplizierte Möglichkeit, sich und Ihre Mitarbeiterlnnen fortzubilden? Dann nutzen Sie doch das PflegeKolleg in HEILBERUFE. Am PflegeKolleg kann jede Pflegekraft teilnehmen, Punkte sammeln oder sich die benötigten Stunden anerkennen lassen. Dazu lesen Sie die verschiedenen Beiträge im PflegeKolleg aufmerksam durch und füllen anschließend den Fragebogen aus. Ambulanten Diensten und Pflegeeinrichtungen bieten wir die Teilnahme zu besonderen Konditionen an: Gern können Sie die gesammelten Fragebögen

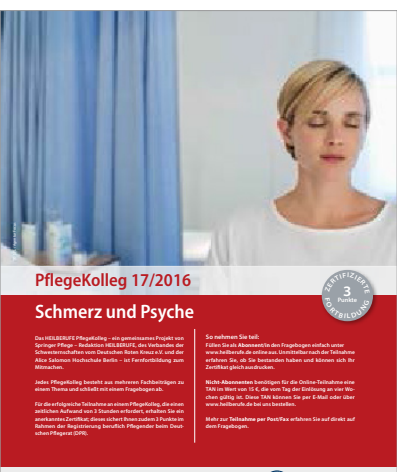

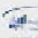
auch per Post oder Fax an uns schicken. Nach der Auswertung erhalten Sie sämtliche Zertifikate in einem Umschlag für Ihre Mitarbeiter zurück. Bezahlt wird - ganz bequem - per Sammelrechnung: eine für alle.

\section{Noch Fragen?}

Rufen oder mailen Sie uns an: Tel.: 030/827875500; E-Mail: heilberufe@springer.com

www.iubh-fernstudium.de

Anerkannt

\section{Fachweiterbildung Notfallpflege an der Charité}

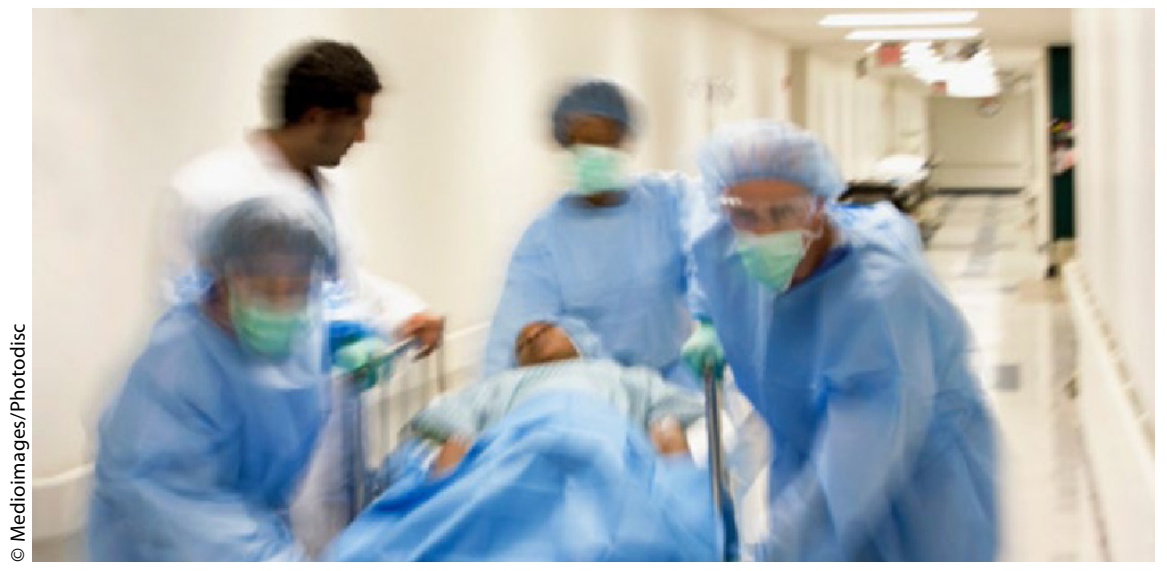

— Die Charité - Universitätsmedizin Berlin bietet eine spezielle Weiterbildung Notfallpflege an. Sie bereitet Pflegekräfte auf die Anforderungen in der Notaufnahme vor. Die deutschlandweit einzigartige Fachweiterbildung ist jetzt staatlich anerkannt. Pflegekräfte dürfen nach erfolgreichem Abschluss der Weiterbildung die geschützte Bezeichnung der Notfallpflegerin oder des Notfallpflegers führen. Inhalte und Umfang der Weiterbildung hat die Senatsverwaltung für Gesundheit und Soziales Berlin in einer Weiterbildungs- und Prüfungsverordnung geregelt. Die Charité setzt mit ihrer Ausbildung, der Prüfung und den damit verbundenen Qualitätsstandards einen Meilenstein in der Notfallpflege in Deutschland. Durch die Weiterbildung von Fachpersonal wird die Qualität in der Patientenversorgung gesichert und weiter verbessert. In der zweijährigen, berufsbegleitenden Fachweiterbildung erhalten die Pflegenden Kenntnisse, Fertigkeiten und Kompetenzen, um auf die Versorgung von Patienten mit akuten medizinischen Notfällen vorbereitet zu sein. Die Weiterbildung umfasst insgesamt 780 Stunden Theorie und 800 Stunden berufspraktische Anteile.
DNQP

\section{Einführung und Anwendung von Expertenstandards}

— Das DNQP hat in diesem Jahr ein neues Weiterbildungsangebot zur Einführung und Anwendung von Expertenstandards gestartet. Ziel ist es, Pflegefachkräfte in Gesundheitsund Pflegeeinrichtungen bei der eigenständigen Implementierung und Anwendung von Expertenstandards aktiver zu unterstützen. Das Weiterbildungsprogramm besteht aus Einzelveranstaltungen zur Einführung und Anwendung von Expertenstandards. Ab dem kommenden Jahr wird außerdem ein Angebot zur unterstützten Einführung eines Expertenstandards angeboten. Das Angebot richtet sich an alle Pflegefachkräfte, die sich in stationären und ambulanten Pflegeeinrichtungen sowie in Krankenhäusern mit der Einführung und Anwendung von Expertenstandards auseinandersetzen.

www.dnqp.de/veranstaltungen 